\title{
Der Triumph der Macher
}

Als wir vor fast 10 Jahren die Zeitschrift FORSCHENDE KomplEMENTÄRMEDIZIN UND KLASSISCHE NATURHEILKUNDE gründeten, sah die Welt ganz anders aus: In der akademischen Medizin stiessen komplementäre Ansätze vor allem auf bitteren Widerstand, doch die Bereitschaft neue Wege zu betreten wuchs. Und gesellschaftlich hatten alle diejenigen Rückenwind, die sich zur Verantwortung für die Natur - und für den Menschen als einem Teil der Natur - bekannten. Das ist heute Vergangenheit.

Die Wende kam bereits Ende der 1980er Jahre mit dem Fall der Berliner Mauer. Da diese Zeit aber nicht nur einen Zusammenbruch der politischen Nachkriegsordnung bedeutete, sondern gleichzeitig einen technologischen Umbruch auch in der Medizin mit sich brachte, dauerte es einige Jahre, bis das Ausmass der Veränderungen deutlich wurde. Für unseren Arbeitsbereich bedeutete es konkret, dass der immer stärker spürbare Einfluss der «virtuellen» Welt den komplementären Ansätzen schrittweise ihre Brisanz nahm.

Für die Arbeit war es zunächst eher positiv, dass der aufreibende Widerstand wegfiel. Weniger positiv war dagegen die Tatsache, dass mit der Brisanz offenbar auch ein Teil der Attraktivität verloren ging. Komplementäre Sichtweisen, die in den 80er Jahren des 20. Jahrhunderts noch als Provokation und daher reizvoll - empfunden wurden, erschienen mittlerweile als eine Möglichkeit unter vielen. Und aus dem Wettbewerb der miteinander konkurrierenden Ansätze sind dann immer eindeutiger diejenigen als Sieger hervorgegangen, die die grösste Machbarkeit versprachen. In der medizinischen Wissenschaft beispielsweise zeigte sich das an den geradezu sakralen Erwartungen, die sich auf die Molekularbiologie richten. Noch nie in der Geschichte der Wissenschaft haben sich die «Macher» so eindeutig gegen die «Denker» durchgesetzt, wie im so genannten Informationszeitalter.

Und so lebt die Welt derzeit auf vielen Ebenen munter im Machbarkeitswahn und kümmert sich wenig um Reflexionen. Nicht das «Warum», sondern das «Wie» beschäftigt gleicher- massen Wissenschaft, Politik und Bürger. Karl Marx hätte sich über diese Vollendung seines dialektischen Materialismus sicher köstlich amüsiert, kam sie doch paradoxerweise zu einem Zeitpunkt, zu dem das endgültige Scheitern seiner Ideen als sicher galt. Die marxistische Sehnsucht nach der technologischen Beherrschung der Welt ging aber nicht mit der Kapitulation des real existierenden Sozialismus unter, sondern hatte sich im global agierenden Kapitalismus besonders fest gesetzt. Mehr Dialektik kann man nun wirklich nicht verlangen.

Von solchen Makroentwicklungen blieb natürlich auch die Medizin nicht unberührt - mit verheerenden Folgen für komplementäre Ansätze und Methoden. Während sich die Sichtweise innerhalb der Wissenschaften immer stärker verengte und das Spezialistentum weiter zunahm, nahm die Dialogfähigkeit ab. Über Ansätze, Methoden und Ziele der Forschung wird daher kaum noch diskutiert, dafür um so mehr über Patente und Rückfinanzierungsmöglichkeiten.

Diese Entwicklung spiegelt sich natürlich auch in unserer Zeitschrift wider. Während wir in den ersten Jahren unserer Existenz vor allem mit dem Problem einer zu großen Praxisferne zu kämpfen hatten, liegt Ihnen mit diesem Heft eine Ausgabe vor, die fast ausschliesslich Arbeiten beinhaltet, die sich mit dem praktischen Alltag beschäftigen. Bis hin zu Überlegungen über Kosten-Nutzen-Analysen einer Behandlung.

So erfreulich es auch ist, Leserwünsche zu erfüllen, so muss ich doch bekennen, dass mich diese Entwicklung nicht nur freut. Denn angetreten sind wir mit dem Ziel, eingefahrene Denkweisen in Frage zu stellen, für mehr konstruktive Unruhe in der medizinischen Wissenschaft zu sorgen und denjenigen Stimmen zu einem Forum zu verhelfen, die gerade wegen ihres unkonventionellen Ansatzes kaum eine Chance haben, sich öffentlich zu artikulieren. Nicht nur Historikern ist es gegenwärtig, dass Triumphe nur Siege auf Zeit sind. Will diese Zeitschrift ihrem ursprünglichen Zweck dienen, muss sie wieder «unbequemer» werden. Helfen Sie uns dabei!

M. Ullmann, München

\section{KARGER}

Fax +497614520714

(c) 2002 S. Karger GmbH, Freiburg

E-mail Information@Karger.de www.karger.com/journals/fkm

www.karger.com 